Marquette University

e-Publications@Marquette

8-3-2012

\title{
The Fe-type Nitrile Hydratase from Comamonas testosteroni Ni1 Does Not Require an Activator Accessory Protein for Expression in Escherichia coli
}

\author{
Misty L. Kuhn \\ Loyola University Chicago \\ Salette Martinez \\ Loyola University Chicago \\ Natalie Gumataotao \\ Loyola University Chicago \\ Uwe Bornscheuer \\ Greifswald University \\ Dali Liu \\ Loyola University Chicago
}

See next page for additional authors

Follow this and additional works at: https://epublications.marquette.edu/chem_fac

Part of the Chemistry Commons

\section{Recommended Citation}

Kuhn, Misty L.; Martinez, Salette; Gumataotao, Natalie; Bornscheuer, Uwe; Liu, Dali; and Holz, Richard C., "The Fe-type Nitrile Hydratase from Comamonas testosteroni Ni1 Does Not Require an Activator Accessory Protein for Expression in Escherichia coli" (2012). Chemistry Faculty Research and Publications. 289.

https://epublications.marquette.edu/chem_fac/289 
Authors

Misty L. Kuhn, Salette Martinez, Natalie Gumataotao, Uwe Bornscheuer, Dali Liu, and Richard C. Holz

This article is available at e-Publications@Marquette: https://epublications.marquette.edu/chem_fac/289 
Marquette University

\section{e-Publications@Marquette}

\section{Chemistry Faculty Research and Publications/College of Arts and Colleges}

This paper is NOT THE PUBLISHED VERSION; but the author's final, peer-reviewed manuscript. The published version may be accessed by following the link in the citation below.

Biochemical and Biophysical Research Communications, Vol. 424, No. 3 (August 3, 2012): 365-370. DOI. This article is (C) Elsevier and permission has been granted for this version to appear in ePublications@Marquette. Elsevier does not grant permission for this article to be further copied/distributed or hosted elsewhere without the express permission from Elsevier.

\section{The Fe-type nitrile hydratase}

\section{from Comamonas testosteroni Ni1 does not require an activator accessory protein for expression in Escherichia coli}

Misty L. Kuhn

Department of Chemistry and Biochemistry, Loyola University Chicago, Chicago, IL Salette Martinez

Department of Chemistry and Biochemistry, Loyola University Chicago, Chicago, IL Natalie Gumataotao

Department of Chemistry and Biochemistry, Loyola University Chicago, Chicago, IL Uwe Bornscheuer Institute of Biochemistry, Department of Biotechnology \& Enzyme Catalysis, Greifswald University, Felix-Hausdorff-Str. 4, 17487 Greifswald, Germany

Dali Liu

Department of Chemistry and Biochemistry, Loyola University Chicago, Chicago, IL

Richard C. Holz 


\section{Abstract}

We report herein the functional expression of an Fe-type nitrile hydratase (NHase) without the co-expression of an activator protein or the Escherichia coli chaperone proteins GroES/EL. Soluble protein was obtained when the $\alpha$ - and $\beta$-subunit genes of the Fe-type NHase Comamonas testosteroni Ni1 (CtNHase) were synthesized with optimized E. coli codon usage and co-expressed. As a control, the Fe-type NHase from Rhodococcus equi TG3282 (ReNHase) was expressed with (ReNHase ${ }^{+A c t}$ ) and without (ReNHase ${ }^{-A c t}$ ) its activator protein, establishing that expression of a fully functional, metallated ReNHase enzyme requires the co-expression of its activator protein, similar to all other Fe-type NHase enzymes reported to date, whereas the CtNHase does not. The X-ray crystal structure of $C t$ NHase was determined to $2.4 \AA ̊$ resolution revealing an $\alpha \beta$ heterodimer, similar to other Fe-type NHase enzymes, except for two important differences. First, two His residues reside in the CtNHase active site that are not observed in other Fe-type NHase enzymes and second, the active site Fe(III) ion resides at the bottom of a wide solvent exposed channel. The solvent exposed active site, along with the two active site histidine residues, are hypothesized to play a role in iron incorporation in the absence of an activator protein.

\section{Graphical abstract}

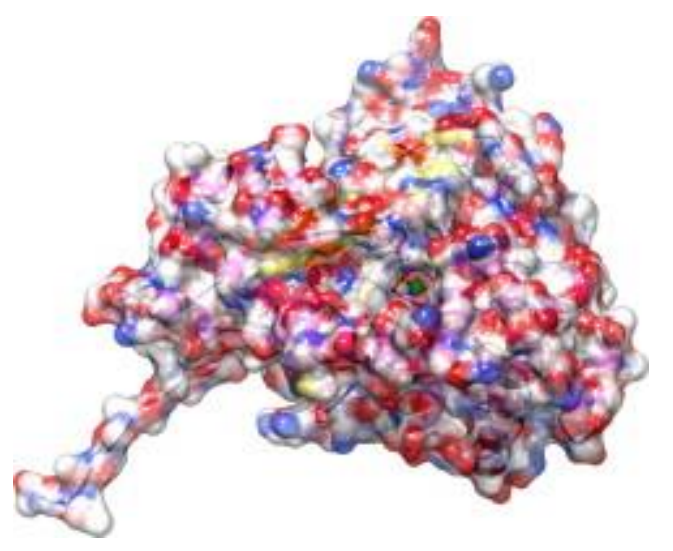

\section{Highlights}

- Functional expression of an Fe-type nitrile hydratase without an activator protein. X-ray structure of the nitrile hydratase from Comamonas testosteroni Ni1 at $2.4 \AA$. Iron active site resides at the bottom of a wide solvent exposed channel. Two His residues reside in the active site that are not conserved. Solvent exposed active site and/or His residues may play a role in iron incorporation.

\section{Keywords}

Nitrile hydratase, Iron, Hydrolysis, X-ray crystallography

\section{Introduction}

Nitrile hydratases (NHase, EC 4.2.1.84) catalyze the hydration of nitriles to their corresponding amides under ambient conditions and physiological pH [1], [2]. NHases have attracted substantial interest as biocatalysts for industrial applications such as the large scale production of acrylamide [3] and nicotinamide [4]. X-ray crystallographic studies have shown that NHases are $\alpha_{2} \beta_{2}$ heterotetramers with an active site consisting of three cysteine residues, two amide nitrogens, a water molecule, and either a non-heme Fe(III) ion (Fe-type) or a non-corrin Co(III) ion (Co-type) [5], [6]. Two of the active site cysteine residues are post-translationally modified 
to cysteine-sulfinic acid $\left(-\mathrm{SO}_{2} \mathrm{H}\right)$ and cysteine-sulfenic acid $(-\mathrm{SOH})$, which yields an unusual metal coordination geometry termed a "claw-setting". Although the structures of Fe- and Co-type NHases are very similar, Fe-type NHases only bind Fe(III) and Co-type NHases only bind Co(III) [5]. Several open reading frames (ORFs) have been identified just downstream from the structural $\alpha$ - and $\beta$-subunit genes in NHases, and one of these genes has been proposed to function as an activator protein [7], [8], [9]. While Co- and Fe-type NHase enzymes share high sequence identity, their respective activator proteins are different sizes and share little sequence identity [10], [11]. The prevailing dogma is that both Co- and Fe-type NHase enzymes require the co-expression of an activator protein to be soluble and fully active [7], [8], [9].

Recently, it was reported that the Fe-type CtNHase, cloned from the Comamonas testosteroni Ni1 genomic DNA could be expressed in Escherichia coli in the absence of an activator protein [12]. The resulting enzyme was insoluble unless the $E$. coli chaperone proteins GroES/EL were co-expressed, in which case soluble enzyme was obtained albeit with low activity. The observation of low activity levels is typical for Fe-type NHases expressed in the absence of an activator protein [5], [13]. To determine whether the limited availability of rare tRNAs for CtNHase expression in E. coli or the lack of an activator protein or molecular chaperone was the true cause of its insolubility and poor activity, the genes encoding the $\alpha$-and $\beta$-subunits of $C t N H$ ase were synthesized with optimized $E$. coli codon usage and heterologously expressed in E. coli. The resulting CtNHase is fully functional as evidenced by the enzyme containing its full complement of $\mathrm{Fe}(\mathrm{III})$ and the equatorial Cys residues being appropriately oxidized, all without the co-expression of an activator protein or the E. coli GroES/EL molecular chaperones. This is the first report of an Fe-type NHase that does not require an activator protein for metal ion insertion or for facilitating the oxidation of the active site cysteine residues.

\section{Materials and methods}

\subsection{Materials}

Acrylonitrile was purchased from Sigma-Aldrich (St. Louis, MO, USA). Synthesized genes and primers were purchased from Integrated DNA Technologies, Inc., (IDT). All other materials were purchased at the highest quality available.

\subsection{C. testosteroni Ni1 nitrile hydratase (CtNHase) plasmid construction}

The $\alpha$ - and $\beta$-subunit genes of $C t N H$ ase were individually synthesized with optimized $E$. coli codon usage and were cloned into the pIDT-SMART kanamycin resistant vector. Protein sequences for the $\alpha$ - and $\beta$ - subunit genes were obtained from Cerbelaud et al. [14]. Optimized sequences for each gene are provided in the Supplemental material (Figs. S1 and S2). Restriction sites were Ncol and HindIII for the $\alpha$-subunit gene and Ndel and Kpnl for the $\beta$-subunit. A polyhistidine $\left(\mathrm{His}_{6}\right)$ affinity tag was engineered onto the $\mathrm{C}$-terminus of the $\beta$ subunit using Phusion DNA polymerase (New England Biolabs) and the following primers: forward 5'-GCA CCC ATA TGG ACG GCA TGC-3' and reverse 5'-GGT ACC TTA ATG ATG ATG ATG ATG GTG CAC CTC TGC G-3'. The polymerase chain reaction (PCR) product was sub-cloned into the $\mathrm{PSC}-\mathrm{B}$-amp/kan vector using the Strataclone Blunt PCR cloning Kit (Stratagene). The $\alpha$ - and $\beta$-C-His 6 subunit genes were sub-cloned into MCSI and MCSII of the kanamycin resistant pCOLADuet-1 expression vector (Novagen), respectively, to create the plasmid pSMCt $\alpha \beta$ His. The sequence was confirmed using automated DNA sequencing at the University of Chicago Cancer Research Center DNA sequencing facility.

\subsection{Rhodococcus equi TG328-2 nitrile hydratase (ReNHase) expression in the presence and absence of an activator protein}

The $R$. equi TG328-2 nitrile hydratase (ReNHase) and activator plasmids were obtained from Bornscheuer et al. [15]. The ReNHase gene was sub-cloned into the ampicillin resistant pET21a+ expression vector and the activator gene into the kanamycin resistant $\mathrm{pET} 28 \mathrm{a}^{+}$expression vector using $\mathrm{Ndel}$ and $\mathrm{HindlI}$ restriction 
sites. ReNHase was expressed in the presence (ReNHase $\left.{ }^{+A c t}\right)$ or absence (ReNHase $\left.{ }^{-A c t}\right)$ of its Fe-type activator protein as previously described [15].

\subsection{CtNHase expression in the absence of an activator protein}

The CtNHase $\alpha$ - and $\beta$-subunits ( $\sim 23$ and $\sim 23.5 \mathrm{kDa}$, respectively) were expressed alone, without co-expressing an Fe-type activator protein or the E. coli chaperones GroES/EL, in the following manner. The plasmid pSMCta $\beta$ His was freshly transformed into BL21(DE3) competent cells (Stratagene), and a single colony was used to inoculate $50 \mathrm{ml}$ of LB-Miller culture containing $50 \mu \mathrm{g} / \mathrm{mL}$ kanamycin with shaking overnight at $37^{\circ} \mathrm{C}$. This culture $(\sim 7 \mathrm{ml})$ was used to inoculate a $1 \mathrm{~L}$ culture and the cells were grown at $37^{\circ} \mathrm{C}$ until the $O D_{600 n m}$ reached $0.6-0.8$. The culture was cooled on ice, induced with $0.1 \mathrm{mM}$ isopropyl $\beta$-d-1-thiogalactopyranoside (IPTG), and expressed at $20^{\circ} \mathrm{C}$ for $16 \mathrm{~h}$. Cells were harvested by centrifugation at $5000 \mathrm{~g}$ and $4{ }^{\circ} \mathrm{C}$ for 5 min in a Beckman Coulter Avanti JA-10 rotor. Cell pellets were resuspended in buffer A (50 mM Na $2 \mathrm{HPO}_{4} \mathrm{pH} 7.5,300 \mathrm{mM} \mathrm{NaCl}$, $10 \mathrm{mM}$ imidazole) at a ratio of $5 \mathrm{ml}$ per gram of cells, then sonicated for $4 \mathrm{~min}$ ( $30 \mathrm{~s}$ on $45 \mathrm{~s}$ off) at $21 \mathrm{~W}$ using a Misonix sonicator 3000 . The crude extract was obtained after centrifugation in a JA-20 rotor at $15,000 \mathrm{~g}$ and $4{ }^{\circ} \mathrm{C}$ for $20 \mathrm{~min}$. The sample was subjected to another round of centrifugation before purification to remove particulate matter.

\subsection{Purification of CtNHase and ReNHase co-expressed with (ReNHase ${ }^{+A c t}$ ) and without activator (ReNHase ${ }^{- \text {Act }}$ )}

Crude extracts of CtNHase, ReNHase ${ }^{+A c t}$, or ReNHase ${ }^{-A c t}$ were loaded onto a $5 \mathrm{ml} \mathrm{Ni-NTA}$ (nitrilotriacetic acid) Superflow Cartridge (Qiagen) for immobilized metal affinity chromatography (IMAC) using an ÄKTA FPLC P-960. The column was washed with four column volumes (CVs) of buffer $A$, and the protein was eluted with a linear gradient (0-100\%) of buffer B (buffer A with $500 \mathrm{mM}$ imidazole) over $20 \mathrm{CVs}$ at a flow rate of $1 \mathrm{ml} / \mathrm{min}$. Active protein fractions were pooled and concentrated with an Amicon Ultra-15 10,000 MWCO centrifugal filter unit (Millipore) resulting in $\sim 10 \mathrm{mg} / \mathrm{L}$ of soluble CtNHase and $\sim 50 \mathrm{mg} / \mathrm{L}$ of ReNHase $^{\text {+Act }}$ (Fig. S3). Protease inhibitors or butyric acid was not required during purification of CtNHase, but $40 \mathrm{mM} n$-butyric acid was included in all buffers for ReNHase purification. For crystallization trials, CtNHase was further purified using a HiLoad 16/60 Superdex 200 prep grade gel filtration column (GE Healthcare). The protein eluted over one CV of buffer C (50 mM HEPES pH 7.5, $300 \mathrm{mM} \mathrm{NaCl}$ ) at a flow rate of $0.5 \mathrm{ml} / \mathrm{min}$. The fractions were pooled, concentrated, and buffer was changed to 50 mM HEPES pH 7.0 using an Amicon Ultra-15 10,000 MWCO centrifugal filter unit.

Purified protein samples were analyzed by SDS-PAGE with a $12.5 \%$ polyacrylamide SPRINT NEXT GEL ${ }^{\text {TM }}$ (Amresco). Gels were stained with Gel Code Blue (Thermo-Fisher Scientific). Protein concentration of crude extracts was determined using a Coomassie (Bradford) Protein Assay Kit (Pierce) and pure protein by measuring the absorbance at $280 \mathrm{~nm}$ with a Nanodrop Spectrophotometer ND1000. Theoretical molecular weights and protein extinction coefficients were calculated with the ExPASy compute $\mathrm{pl} / \mathrm{Mw}$ tool. The molecular weight for the $C t N H a s e$ tetramer was $92,828 \mathrm{~g} / \mathrm{mol}$ with an extinction coefficient of $118,720 \mathrm{~cm}^{-1} \mathrm{M}^{-1}$. For the ReNHase tetramer, a molecular weight of $97,888 \mathrm{~g} / \mathrm{mol}$ was calculated with an extinction coefficient of $149,660 \mathrm{~cm}^{-1} \mathrm{M}^{-1}$. These molecular weights are in good agreement with SDS-PAGE data.

\subsection{Kinetic analysis of CtNHase, ReNHase ${ }^{+A c t}$, and ReNHase ${ }^{-A c t}$}

The enzymatic activity of $C t N H a s e$, ReNHase ${ }^{+A c t}$, and ReNHase $^{-A c t}$ towards acrylonitrile ( $225 \mathrm{~nm}, \varepsilon=2.9 \mathrm{mM}^{-1} \mathrm{~cm}^{-1}$ ) was measured using a Shimadzu UV-2450 spectrophotometer. A $1 \mathrm{ml}$ reaction consisted of $50 \mathrm{mM}$ Tris-HCl buffer pH 7.5 (CtNHase) or $50 \mathrm{mM}$ phosphate buffer pH 7.5 (ReNHase ${ }^{+A c t}$ and ReNHase ${ }^{-A c t}$ ) and various concentrations of acrylonitrile. All assays were performed at room temperature and data analysis was performed using Origin Lab 7.5. The kinetic constants $V_{\max }$ and $K_{\mathrm{m}}(k)$ were 
calculated using the equation $\left[y=V_{0}+\left(V_{\max }-V_{0}\right)\left(x^{n} /\left(k^{n}+x^{n}\right)\right)\right]$ where $n=1$ and $V_{0}$ is zero to give the equation $y=V_{\max } *(x /(k+x))$. One Unit is defined as $1 \mu \mathrm{mol} / \mathrm{min}$.

\subsection{Metal analysis}

Protein samples for CtNHase $(0.15 \mathrm{mg} / \mathrm{ml}), \operatorname{ReNHase}^{\text {AAct }}(0.15 \mathrm{mg} / \mathrm{ml})$, and ReNHase ${ }^{-A c t}(0.19 \mathrm{mg} / \mathrm{ml})$ were digested with concentrated nitric acid at $70{ }^{\circ} \mathrm{C}$ for $10 \mathrm{~min}$ and then cooled to room temperature. These samples were diluted to $5 \mathrm{ml}$ total volume with deionized water to give a final nitric acid concentration of $5 \%$, and were filtered using $0.2 \mu \mathrm{m}$ Supor membrane syringe filters (Pall). A nitric acid blank was also prepared. The samples were analyzed using inductively coupled atomic emission spectroscopy (ICP-AES) at the Integrated Molecular Structure Education and Research Center (IMSERC) at Northwestern University (Evanston, IL, USA).

\subsection{CtNHase crystallization and data collection}

Crystals of $\mathrm{CtNHase}$ were obtained by the hanging drop vapor diffusion method. The initial sparse matrix screen was carried out using a Gryphon crystallization robot from Art Robbins Inc. with commercial screen sets including Wizard Screen I-IV from Emerald Biosystems (Bainbridge Island, WA, USA) and Crystal Screen I-II, Peg Ion Screen, Index Screen I-II, and Crystal Screen Cryo from Hampton Research. Once initial crystallization conditions were obtained, they were optimized manually by varying all component concentrations. Optimized conditions were: $1 \mu \mathrm{l}$ of purified CtNHase $(15,20$, or $30 \mathrm{mg} / \mathrm{ml})$ in $50 \mathrm{mM} \mathrm{HEPES} \mathrm{pH} 7.0$ with an equal volume of the crystallization reservoir solution (1.08 $\mathrm{M} \mathrm{K}_{2} \mathrm{HPO}_{4}, 0.49 \mathrm{M} \mathrm{NaH}_{2} \mathrm{PO}_{4}$ with $25 \%$ or $30 \%$ (v/v) glycerol). The best quality $\mathrm{CtNHase}$ crystals were obtained at $20{ }^{\circ} \mathrm{C}$ after 5 days. For X-ray data collection, the crystals were soaked in the reservoir solution with $30 \%(\mathrm{v} / \mathrm{v})$ glycerol as a cryo-protectant before flash freezing in liquid nitrogen. Data sets were collected at the SBC 19-ID beamline at the Advanced Photon Source, Argonne National Laboratory (Argonne, IL, USA). Monochromatic data collection was conducted at a wavelength of $0.98 \AA$ using a Quantum 315 CCD detector providing a data set with a resolution to $2.4 \AA$.

\subsection{Structure determination and refinement}

The X-ray data set for CtNHase was indexed, integrated and scaled using HKL3000 software and the statistics revealed that the data were of good quality (Table 1) [16]. The space group was $P 3_{1}$ with eight copies of $C t$ NHase heterodimers in each asymmetric unit giving a solvent content of $71 \%$. Molecular replacement was carried out with a known NHase structural model (PDB ID: 1AHJ) [17] using the program Phaser from the CCP4 software suite. Once a solution was obtained, model building was conducted in COOT [18]; rigid-body refinement and restrained refinement was conducted in refmac5 [19]. In order to remove model bias and achieve the best refinement result possible, simulated annealing refinement, TLS refinement, and ordered solvent identification were conducted using PHENIX.refine [20] while model building continued until the lowest $R_{\text {free }} / R$ values were achieved. Active site metal ion occupancies were initially set at one resulting in negative difference electron density $\left(F_{0}-F_{c}\right.$ map) around the metal center (not shown). Metal analyses indicated $\sim 0.8$ equivalents of iron per heterodimer, therefore, the final structural model was refined with iron ion occupancies and those of the three coordinating residues (CSA102, S103 and CSA104) set at 0.8 . Positive difference electron density $\left(F_{\mathrm{o}}-F_{\mathrm{c}}\right.$ map) around this region was observed (not shown) after refinement, which is likely due to the contribution of residues 102-104 from the 20\% apo-enzyme molecules in the crystal. The structure was refined in PHENIX with the completed model possessing $R_{\text {free }} / R$ values of $22.7 / 18$.8. Since the $P 3_{1}$ space group can be indicative of twining, the $L$-test for twining was conducted using the Xtriage program in PHENIX revealing that the intensity statistics behave as expected, indicating no twining.

Table 1. Data and Refinement Statistics.

\begin{tabular}{|l|l|}
\hline PDB code & xxxx \\
\hline Resolution range, $\AA$ & $36.2-2.4$ \\
\hline
\end{tabular}




\begin{tabular}{|c|c|}
\hline Space group & $P 3_{1}$ \\
\hline \multicolumn{2}{|l|}{ Cell dimensions } \\
\hline$a, b, c, \AA$ & $111.4,111.4,475.3$ \\
\hline$n_{1}^{\circ}$ & $90,90,120$ \\
\hline Total (unique) reflections & $128,0741(262,123)$ \\
\hline Completeness, $\%$ & $99.5(99.2)$ \\
\hline Linear $R_{\text {merge }}{ }^{a}$, \% & $13.3(60.2)$ \\
\hline I/ (I/ cut-off $)$ & $11.0(1.0)$ \\
\hline$R_{\text {crys }}{ }^{\mathrm{b}} / R_{\text {free }}{ }^{\mathrm{c}}, \%$ & $18.8 / 22.7$ \\
\hline rmsd $^{d}$ bond, $\AA$ & 0.01 \\
\hline rmsd $^{d}$ angle, ${ }^{\circ}$ & 1.4 \\
\hline Average $\mathrm{B}, \AA^{2}$ & 47.8 \\
\hline Estimated coordinates error, $\AA$ & 0.29 \\
\hline
\end{tabular}

a Linear $R_{\text {merge }}=\Sigma\left|I_{\text {obs }}-l_{\text {avg }}\right| / \Sigma l_{\text {avg }}$.

b $R_{\text {crys }}=\Sigma\left|F_{\text {obs }}-F_{\text {calc }}\right| / \Sigma F_{\text {obs }}$.

c Five percent of the reflection data were selected at random as a test set and only these data were used to calculate $R_{\text {free. }}$.

$\mathrm{d}$ rmsd, root mean square deviation.

\section{Results and discussion}

\subsection{Kinetic characterization of CtNHase, ReNHase ${ }^{+A c t}$, and ReNHase ${ }^{-A c t}$}

$C t$ NHase was expressed in E. coli without the co-expression of the E. coli molecular chaperones GroES/EL or an activator protein but with $E$. coli codon usage and a hexahistidine tag on the $C$-terminus of the $\beta$-subunit. Expression of $\mathrm{CtNHase}$ and purification using IMAC resulted in $\sim 10 \mathrm{mg} / \mathrm{L}$ of soluble $\mathrm{CtNH}$ ase enzyme. Kinetic characterization of $C t$ NHase using acrylonitrile as the substrate provided a $k_{\text {cat }}$ of $71 \pm 5 \mathrm{~s}^{-1}$ and a $K_{\mathrm{m}}$ of $350 \pm 50 \mathrm{mM}$. Since the turnover rate is more than 7-fold higher than any previous report for CtNHase [12], [13], [21], it was hypothesized that the metal ion was properly inserted and both active site Cys residues were properly oxidized in the absence of an activator protein, which is required for full enzymatic activity [22]. For comparison purposes, the Fe-type ReNHase was co-expressed with its activator protein (ReNHase $\left.{ }^{+A c t}\right)$, purified, and kinetically characterized. Using acrylonitrile as the substrate, the enzyme exhibited a $k_{\text {cat }}$ of $2400 \mathrm{~s}^{-1}$ and a $K_{\mathrm{m}}$ of $4.3 \pm 1.7 \mathrm{mM}$, which is in good agreement with previous studies [15]. Although expression of ReNHase ${ }^{-A c t}$ produced soluble protein, no enzymatic activity was observed, most likely due to its inability to incorporate metal in the absence of an activator protein.

\subsection{Metal analysis and spectral characterization of CtNHase and ReNHase}

A combination of UV-vis spectroscopy and metal analysis was used to determine if CtNHase, ReNHase ${ }^{+A c t}$ and ReNHase ${ }^{-A c t}$ contained their full complement of Fe(III). After purification, the CtNHase and ReNHase ${ }^{+A c t}$ enzymes exhibited an emerald green color, whereas the ReNHase ${ }^{\text {-Act }}$ was colorless. The UV-vis spectra for CtNHase and ReNHase ${ }^{+A c t}$ show the characteristic $\mathrm{S} \rightarrow \mathrm{Fe}$ (III) ligand-to-metal-chargetransfer (LMCT) band at $\sim 700 \mathrm{~nm}\left(\varepsilon=\sim 6000 \mathrm{~cm}^{-1} \mathrm{M}^{-1}\right.$ and $3500 \mathrm{~cm}^{-1} \mathrm{M}^{-1}$, respectively), however no absorbance was detected for ReNHase ${ }^{-A c t}$ (Fig. 1). ICP-AES data indicate that CtNHase contained $\sim 1.6$ irons per $\alpha_{2} \beta_{2}$ heterotetramer, ReNHase ${ }^{+A c t}$ had $\sim 1.7$ equivalents of iron per $\alpha_{2} \beta_{2}$ heterotetramer, while no iron was detected for ReNHase ${ }^{-A c t}$. These data clearly indicate that ReNHase strictly requires the co-expression of its corresponding Fe-type activator for an active enzyme and the presence of an affinity tag plays no role in metal incorporation. They also confirm that CtNHase contains its full complement of Fe(III) metal and can thus be 
expressed in E. coli in the absence of an Fe-type NHase activator protein or the E. coli molecular chaperones GroES/EL.

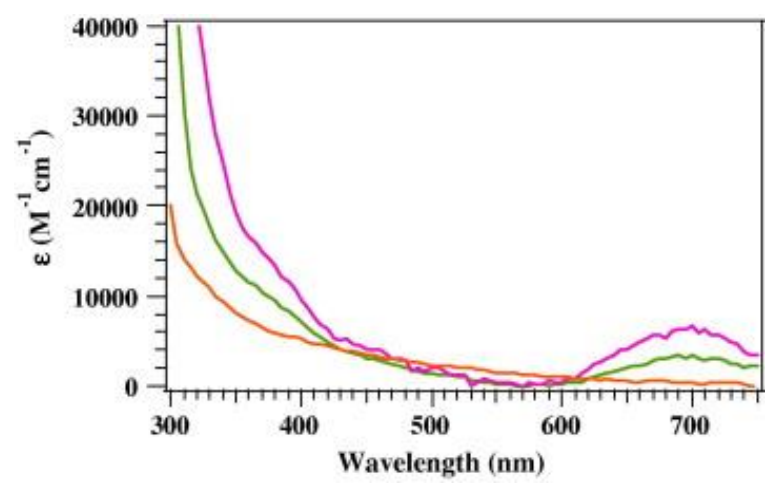

Fig. 1. UV-visible spectra of CtNHase Ni1 (-), ReNHase TG328-2 (-) co-expressed with activator, and ReNHase TG328-2 co-expressed without activator (-).

\subsection{Crystal structure of CtNHase}

The three-dimensional X-ray crystal structure of $C$ tNHase was determined to $2.4 \AA$ resolution. The structure verifies that the recombinant $\mathrm{CtNH}$ ase binds its full complement of iron and, in general, is structurally similar to other Fe-type NHase enzymes (Fig. 2). The $\alpha$-subunit of $\mathrm{CtNHase}$ is embedded in the center of the $\beta$-subunit, while the $\mathrm{N}$-terminus of the $\alpha$-subunit forms a helix that is embedded among three helices from the $\beta$-subunit (Fig. 2A). Supported by ICP-AES data, we set the occupancies of the Fe(III) ion and its coordination residues at 0.8 during structural refinement resulting in satisfactory electron density maps and good $R_{\text {free }} / R$ values. The $\mathrm{Fe}(\mathrm{III})$ ions in CtNHase are five coordinate and bound by C99, C102, and C104 as well as two backbone amide nitrogens, where C102 and C104 are both oxidized to cysteine-sulfinic acids (CSA) (Fig. 2B). The full oxidation of the equatorial Cys residues is expected since crystals of $\mathrm{CtNHase}$ were obtained under aerobic conditions over the course of a week. Two amide nitrogen atoms and the oxidized sulfur atoms are roughly in the same plane as the metal ion. The sulfur atom from C99 forms a coordination bond roughly perpendicular to this plane, which results in a slightly distorted square-based pyramidal geometry. On the opposite side of C99, no sixth ligand such as NO or a water molecule is present as is often observed for Fe-type NHase enzymes [5], which is possibly due to the resolution of the present structure. A phosphate ion is present in the active site, likely due to phosphate $(1.57 \mathrm{M})$ in the crystallization buffer (Fig. 2B). This phosphate ion forms hydrogen bonds with $\alpha-\mathrm{H} 80(2.6 \AA)$ and $\alpha-\mathrm{H} 81$ (2.8 $\AA$ ) along with $\alpha-\mathrm{R} 157$ (2.9 and $2.8 \AA$ from two nitrogen atoms, respectively) and an oxygen atom of CSA104 (2.6 $\AA$ ). Interestingly, these histidine residues are not strictly conserved in the $\alpha$-subunits of Fe-type NHase enzymes (Fig. S4). 
A
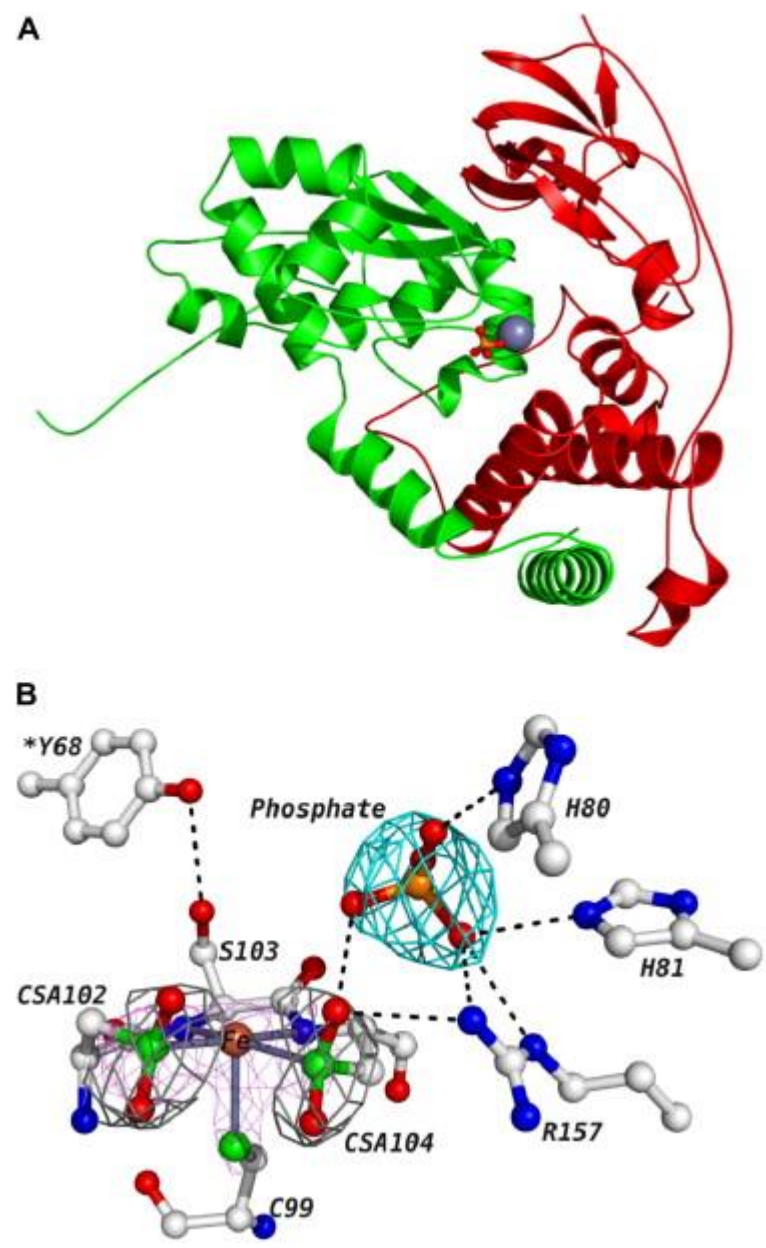

Fig. 2. Structure of the $C t$ NHase heterodimer. (A) The $\alpha$-subunit is in green and $\beta$-subunit is in red. The iron ion is shown as a grey sphere and the phosphate ion bound to the active site is shown in ball-and-stick form.

(B) CtNHase active site containing a phosphate ion in ball-and-stick form, the iron ion is shown as an orange sphere, bonds are shown as silver sticks, and hydrogen bonds are shown as dashed lines. The asterisk indicates that $Y 68$ is from the $\beta$-subunit. Electron density maps $\left(2 F_{0}-F_{c}\right)$ are shown around key chemical groups: the two sulfinic acid groups are shown in grey at $1.2 \sigma$ while the maps around the heavy atom are shown in magenta

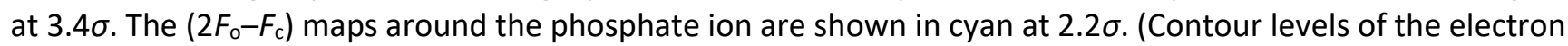
density maps are adjusted for both validation and clarity.).

Structural comparison of the CtNHase model and the Fe-type NHase from Rhodococcus sp. 312 (1AHJ) [17], which exhibits $\sim 50 \%$ identity to CtNHase, indicates that the two structures are very similar with a core rmsd (root mean square deviation) of $1.13 \AA$. Three regions of the CtNHase are different from 1AHJ (Fig. 3A). These include the $\mathrm{C}$-terminal region of the $\alpha$-subunit, the $\mathrm{N}$-terminus of the $\alpha$-subunit, and a loop region (76-83) of $\alpha$ subunit just above the $\mathrm{Fe}(\mathrm{III})$ active site. Neither this loop region in CtNHase nor the equivalent loop region (88$94)$ in $1 \mathrm{AHJ}$ is involved in any crystallographic contact. It is therefore unlikely that the structural differences are caused by mildly different crystallization conditions ( $\mathrm{pH} 7$ vs. $\mathrm{pH}$ 8.5). Alteration of this loop region above the active site results in the formation of an unusually wide-open solvent accessible active site (Fig. 3B). This accessibility provides a direct approach for the substrate to the active site and coincides directly with the unfilled axial position of the Fe(III) ion (Fig. 3B). Typically, the trivalent metal ions of both Co- and Fe-type NHase enzymes are buried and accessible only through narrow channels [5]. The solvent inaccessibility of the trivalent metal ions in NHases has been implicated in observed substrate specificities, as well as the difficulty in preparing the apo- or metal substituted forms of NHases [5]. In the contrast, the narrow substrate channel observed for 
$1 \mathrm{AHJ}$ requires that the substrate approach the metal center at a $60^{\circ}$ angle off of the axial position (Fig. $3 \mathrm{C}$ ). In $1 \mathrm{AHJ}$, the side chains of $\alpha-Y 40, \alpha-\mathrm{W} 44, \alpha-\mathrm{W} 118, \beta-Y 37, \beta-M 40$ and $\beta-F 41$ block a direct axial approach to the $\mathrm{Fe}(\mathrm{III})$ ion. None of the above residues are conserved and all are significantly bulkier than the spatially related residues in CtNHase.

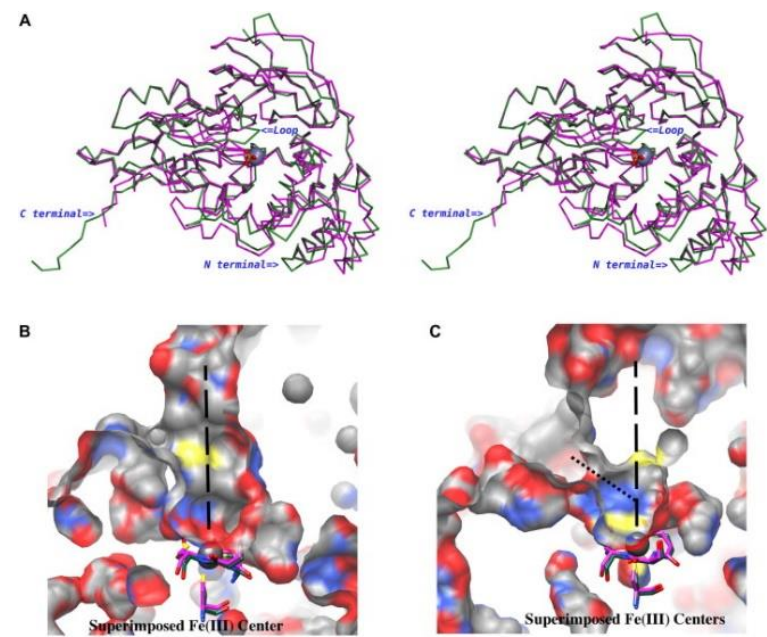

Fig. 3. Structural Comparison of CtNHase and the Starting Model 1AHJ. (A) A wall-eyed stereo display of the superposition of $\mathrm{CtNH}$ ase and $1 \mathrm{AHJ}$. The $\mathrm{CtNH}$ ase structure is in green and the $1 \mathrm{AHJ}$ model is in magenta. Three regions with pronounced differences in the protein backbones occur between the two $\alpha$-subunits and are indicated in the $\mathrm{CtNHase}$ structure with labels at the two terminal regions and a loop region (76-83) above the metal center. (B) Surface representation of the CtNHase heterodimer showing the solvent accessible channel with the active site iron as a grey sphere. The Fe(III) centers of $\mathrm{CtNH}$ ase and $1 \mathrm{AHJ}$ are superimposed. The metal ligands displayed are shown in stick form; the carbon atoms for the residues in CtNHase are green; the carbon atoms of the residues in $1 \mathrm{AHJ}$ are blue. The Fe(III) ion in CtNHase is shown as a silver sphere. A dashed line indicates the axial direction of the metal constellation. (C) Surface representation of 1AHJ showing the solvent accessible channel. The superimposed Fe(III) centers are show as in B.

In conclusion, the construct reported herein for $\mathrm{CtNHase}$ is the first functional expression system for an Fe-type NHase that does not require an NHase activator protein or the $E$. coli chaperone proteins GroEL/ES. These data are contrary to the accepted view regarding the strict requirement of co-expressing NHases with activator proteins for fully active enzymes. Based on the X-ray crystallographic data CtNHase binds it full complement of $\mathrm{Fe}(\mathrm{III})$ ions in its active site and the equatorial Cys residues are appropriately oxidized, which is a requirement for full enzymatic activity. Both metal ion insertion and Cys oxidation are functions previously ascribed to the NHase activator protein. The structure of $\mathrm{CtNH}$ ase reveals that the $\mathrm{Fe}(\mathrm{III})$ ion in the active site is solvent exposed and resides at the bottom of an open cavity. This exposed active site not only provides a much more direct route for substrates, but also for metal ion incorporation. It is possible that the solvent accessibility of the CtNHase active site, both the large opening and the axial approach direction, provides the necessary access for metal ion incorporation without the use of an activator protein. Moreover, the two non-conserved His residues that reside near the active site Fe(III) ion at the bottom of the solvent exposed channel, may play a role in assisting in the incorporation of iron ions into the active site.

\section{Appendix A. Supplementary data}

\section{Download : Download Acrobat PDF file (99KB)}

Supplementary Figures. 


\section{References}

[1] P.K. Mascharak. Structural and functional models of nitrile hydratase. Coord. Chem. Rev., 225 (2002), pp. 201-214

[2] I. Artaud, S. Chatel, A.S. Chauvin, D. Bonnet, M.A. Kopf, P. Leduc. Nitrile hydratase and related non-heme iron sulfur complexes. Coord. Chem. Rev., 190-192 (1999), pp. 577-586

[3] T. Nagasawa, H. Yamada. Microbial production of commodity chemicals. Pure Appl. Chem., 67 (1995), pp. 1241-1256

[4] T. Nagasawa, C.D. Mathew, J. Mauger, H. Yamada. Nitrile hydratase-catalyzed production of nicotinamide from 3-cyanopyridine in Rhodococcus rhodochrous J1. Appl. Environ. Microbiol., 54 (1988), pp. 17601766

[5] J.A. Kovacs. Synthetic analogues of cysteinate-ligated non-heme iron and non-corrinoid cobalt enzymes. Chem. Rev., 104 (2004), pp. 825-848

[6] T.C. Harrop, P.K. Mascharak. Fe(III) and Co(III) centers with carboxamido nitrogen and modified sulfur coordination: lessons learned from nitrile hydratase. Acc. Chem. Res., 37 (2004), pp. 253-260

[7] M. Nishiyama, S. Horinouchi, M. Kobayashi, T. Nagasawa, H. Yamada, T. Beppu. Cloning and characterization of genes responsible for metabolism of nitrile compounds from Pseudomonas chlororaphis B23. J. Bacteriol., 173 (1991), pp. 2465-2472

[8] Y. Hashimoto, M. Nishiyama, S. Horinouchi, T. Beppu. Nitrile hydratase gene from Rhodococcus sp. N-774 requirement for its downstream region for efficient expression. Biosci. Biotechnol. Biochem., 58 (1994), pp. $1859-1869$

[9] M. Nojiri, M. Yohda, M. Odaka, Y. Matsushita, M. Tsujimura, T. Yoshida, N. Dohmae, K. Takio, I. Endo. Functional expression of nitrile hydratase in Escherichia coli: requirement of a nitrile hydratase activator and post-translational modification of a ligand cysteine. J. Biochem., 125 (1999), pp. 696-704

[10] R.A. Cameron, M. Sayed, D.A. Cowan. Molecular analysis of the nitrile catabolism operon of the thermophile Bacillus pallidus RAPc8. Biochim. Biophys. Acta (BBA) - Gen. Subj., 1725 (2005), pp. 35-46

[11] Z. Zhou, Y. Hashimoto, T. Cui, Y. Washizawa, H. Mino, M. Kobayashi. Unique biogenesis of high-molecular mass multimeric metalloenzyme nitrile hydratase: intermediates and a proposed mechanism for selfsubunit swapping maturation. Biochemistry, 49 (2010), pp. 9638-9648

[12] J.M. Stevens, N. Rao Saroja, M. Jaouen, M. Belghazi, J.-M. Schmitter, D. Mansuy, I. Artaud, M.-A. Sari. Chaperone-assisted expression, purification, and characterization of recombinant nitrile hydratase NI1 from Comamonas testosterone. Protein Expr. Purif., 29 (2003), pp. 70-76

[13] M.-A. Sari, M. Jaouen, N.R. Saroja, I. Artaud. Influence of cobalt substitution on the activity of iron-type nitrile hydratase: are cobalt type nitrile hydratases regulated by carbon monoxide? J. Inorg. Biochem., 101 (2007), pp. 614-622

[14] E. Cerbelaud, S. Levy-Schil, D. Petre, F. Soubrier. Novel nitrile hydratases from Comamonas testosteroni and the genes encoding them and their uses. Rhone-Poulenc Chimie SA (1995)

[15] K. Rzeznicka, S. Schätzle, D. Böttcher, J. Klein, U. Bornscheuer. Cloning and functional expression of a nitrile hydratase (NHase) from Rhodococcus equi TG328-2 in Escherichia coli, its purification and biochemical characterization. Appl. Microbiol. Biotechnol., 85 (2010), pp. 1417-1425

[16] Z. Otwinowski, W. Minor. Processing of X-ray diffraction Data Collected in Oscillation Mode. C.W. Carter Jr., R.M. Sweet (Eds.), Methods in Enzymology, Macromolecular Crystallography, part A, Vol. 276, Academic Press, New York (1997), pp. 307-326

[17] W. Huang, J. Jia, J. Cummings, M. Nelson, G. Schneider, Y. Lindqvist. Crystal structure of nitrile hydratase reveals a novel iron centre in a novel fold. Structure, 15 (1997), pp. 691-699

[18] P. Emsley, K. Cowtan. Coot: model-building tools for molecular graphics. Acta Crystallogr. D Biol. Crystallogr., 60 (2004), pp. 2126-2132

[19] G.N. Murshudov, A. Vagin, J. Dodson. Refinement of macromolecular structures by the maximumlikelihood method. Acta Crystallogr. D, 53 (1997), pp. 240-255

[20] P.D. Adams, R.W. Grosse-Kunstleve, L.-

W. Hung, T.R. loerger, A.J. McCoy, N.W. Moriarty, R.J. Read, J.C. Sacchettini, N.K. Sauter, T.C. Terwilliger. 
PHENIX: building new software for automated crystallographic structure determination. Acta Crystallogr. D, 58 (2002), pp. 1948-1954

[21] S.N. Rao, R.C. Holz. Analyzing the catalytic mechanism of the Fe-Type nitrile hydratase from Comamonas testosteroni Ni1. Biochemistry, 47 (2008), pp. 12057-12064

[22] T. Arakawa, Y. Kawano, Y. Katayama, H. Nakayama, N. Dohmae, M. Yohda, M. Odaka. Structural basis for catalytic activation of thiocyanate hydrolase involving metal-ligated cysteine modification. J. Am. Chem. Soc., 131 (2009), pp. 14838-14843

This work was supported by the National Science Foundation (CHE-1058357, RCH) and the ACS Petroleum Research Fund (ACS PRF 50033-ND4, RCH). 